\title{
SINNGEHALTE DER AUFKLÄRUNGSIDEE IN DER PHILOSOPHIE VON NOVALIS
}

\section{Honorata Jakuszko}

Der Artikel bildet eine auf Quellentexten fundierte Rekonstruktion der Weise, auf welche Novalis als Vertreter der deutschen Frühromantik den Begriff der Aufklärung versteht. Seiner Meinung nach sei die Aufklärung mit dem historischen Prozess oder mit der Bildungsgeschichte identisch, deren Ziel die allseitige Entwicklung aller Neigungen der Menschlichkeit sei. Enger gefasst kann die Aufklärung auf das 18. Jahrhundert bezogen werden, das als Epoche der Aufklärung bezeichnet wurde. Novalis macht auf die historischen Prämissen aufmerksam, die den Unterschied zwischen der französischen und der deutschen Aufklärung bedingen ( $u$. a. die Reformation in Deutschland und die Französische Revolution). Als Anfang der neuen und höheren Etappe der Aufklärung in Deutschland betrachtet er die transzendentale Philosophie von I. Kant und J. G. Fichte, die Weimarer Klassik von J. W. Goethe und F. Schiller und die historische Philosophie von F. Schlegel - dem Vertreter der deutschen Frühromantik.

Schlüsselworte: Aufklärungsprogramm, Variante der Aufklärung, deutsche Frühromantik, Novalis, Bildungsgeschichte

\section{Einführende Bemerkungen}

In den philosophischen Diskussionen der letzten Jahrzehnte des 18. Jhs über die Aufklärungsidee sind zwei Einstellungen zu verzeichnen. Die eine betrachtete die Aufklärung als eine zeitgenössische Formation und grenzte sie scharf von den früheren Stadien der sog. Unmündigkeit des Verstandes ab, die andere dagegen behauptete, jede Epoche besitze ihre eigene Aufklärung, ihr goldenes Zeitalter, ihren eigenen Glücksmaß$\operatorname{tab}^{1}$.

HONORATA JAKUSZKO, doktor habilitowany, profesor nadzwyczajny, Instytut Filozofii UMCS w Lublinie, Polska; adres do korespondencji: Pl. Mari CurieSkłodowskiej 4, 20-031 Lublin. E-mail: honjakuszko@wp.pl

${ }^{1}$ Siehe J. G. Herder, Auch eine Philosophie der Geschichte zur Bildung der Menschheit, Suhrkamp, Frankfurt am Main 1967, S. 38, 45, 106. Die erwähnte Unterschiede sind insbesondere im Zusammenhang mit dem Problem des Mittelalters zum Vorschein gekommen. 
Die zweitgenannte These enthält eine Doppeldeutigkeit, sie konnte nämlich als Protest gegen den Fortschrittsgedanken mit der gleichzeitigen Rehabilitation bestimmter Geschichtsperioden verstanden werden, die die Philosophen als dunkel und barbarisch bezeichneten, sie konnte aber mit diesem Gedanken vollkommen einverstanden sein. In einem solchen Fall wäre die Aufklärung als ein Prozess begriffen, in dessen Ergebnis die Menschheit auf verschiedenen Wegen ihre Selbsterkenntnis und eigene Reife anstrebt. In einem anderen Sinne wiederum bedeutete die Aufklärung einen erwünschten Zustand, ein Ideal der Geschichte, wobei es für die einen Denker ein Synonym der Verwirklichung des Verstandes bedeutete, für die anderen dagegen Erfüllung aller Veranlagungen der Menschheit.

Diese zweite Variante war für die deutsche Denkweise besonders charakteristisch, die sich ihrer Eigenständigkeit, ja Überlegenheit vor der französischen Kultur bewusst war, in der eine Verflachung der Inhalte erfolgt habe, die das Aufklärungsprogramm enthielt. Dies kann man bereits in den Schriften der deutschen Aufklärung, z. B. bei Herder in den Aussagen von Goethe und Schiller, auch bei den Romantikern (F. Schlegel und Novalis) verfolgen. Es ist zu bemerken, dass solche Urteile im Klima der Ereignisse der Französischen Revolution formuliert wurden, die man als eine belehrende Geschichtslektion betrachtete, als eine Erfahrung, die viele gängige Losungen revidiert hat, auf die sich das sog. Spießbürgerbewusstsein stützte.

Demnach kann man besonders die letzten Jahrzehnte des 18. Jhs für eine Periode erachten, in der die deutschen Intellektuellen, die manchmal von französischen Anregungen beeinflusst waren, ihren Minderwertigkeitskomplex $\mathrm{zu}$ überwinden und die Dämmerung des französischen Geistes in der Bildungsgeschichte zu begründen begonnen haben. Somit begründeten sie auch die Übernahme der Vormachtstellung in diesem Bereich durch Deutschland. Als Beispiel sein etwa Goethes Formulierung zu erwähnen ${ }^{2}$; nach der der intellektuelle Schwerpunkt in der deutschen Nation liege, oder die Aussage von F. Schlegel, der meinte, die Götter der Deutschen hießen nicht Wotan und Hermann,

\footnotetext{
${ }^{2}$ Siehe Novalis, Schriften. Die Werke Friedrich von Hardenbergs, hrsg. von P. Kluckhohn und R. Samuel unter Mitarbeit von H. Ritter, G. Schulz, H. J. Mähl, Dritte, nach den Handschriften ergänzte, erweiterte und verbesserte Auflage, Bd. I-VI (VI/1-3), W. Kohlhammer Verlag, Stuttgart 1960-2006. Die These Goethes aus seinen Wilhelm Meisters Lehrjahre erwähne ich nach Novalis Schriften, Bd. II, Vermischte Bemerkungen, S. 121, Blütenstaub, S. 107.
} 
sondern Wissenschaft und Kunst ${ }^{3}$. Äußerst klar drückt diese Überzeugung Novalis aus, indem er schreibt: „Der Deutsche ist lange das Hänschen gewesen. Er dürfte aber wohl bald der Hans aller Hänse werden. Er geht ihm, wie es viele dummen Kindern gehn soll: er wird leben und klug sein, wenn seine frühklugen Geschwister längst vermodert sind, und er nun allein Herr im Hause ist"

Daraus ist zu schließen, dass in der Weltgeschichte nicht von einem undifferenzierten Subjekt die Rede sein kann, das das Menschengeschlecht sein sollte. Novalis ist überzeugt, dass es viele Subjekte, viele Persönlichkeiten gibt, die in verschiedenen Perioden Geistesreife erreichen und ihre Leistungen könnte man als eine Reihe darstellen, in der die aufeinanderfolgenden Glieder, dank den Fehlern der Vorgänger bereichert, immer gründlicher und vollkommener die Aufklärungsidee verwirklichen. Für ihr schnelles Entwicklungstempo und verfrühte Reife bezahlt die französische Kultur den Tribut und zwar ist es die Oberflächlichkeit der Geistesbildung, zu früh alternde Ideen, die in der Gesellschaftspraxis bankrott werden. Deutschland dagegen schreibt Novalis - ,geht einen langsamen aber sichern Gang vor den übrigen europäischen Ländern voraus. Während diese durch Krieg, Spekulation und Parthey-Geist beschäftigt sind, bildet sich der Deutsche mit allem Fleiss zum Genossen einer höhern Epoche der Cultur und dieser Vorschritt muss ihm ein grosses Uebergewicht über die Anderen im Lauf der Zeit geben"5.

\section{Die französische und die deutsche Variante der Aufklärung laut Novalis}

Novalis unterscheidet deutlich die französische und die deutsche Variante der Aufklärung, indem er sie entsprechend mit der eng und weit begriffenen Aufklärung identifiziert ${ }^{6}$. Wir versuchen nun zu verfolgen,

\footnotetext{
${ }^{3}$ F. Schlegel, Ideen, [in:] Athenaeum. Eine Zeitschrift von August Wilhelm Schlegel und Friedrich Schlegel, Berlin 1798-1800, Nachdruck Darmstadt, Bd. III, S. 28.

${ }^{4}$ Novalis, Schriften, Bd. II, Vermischte Bemerkungen, S. 60, Blütenstaub, S. 61.

${ }^{5}$ Derselbe, Schriften, Bd. III, Christenheit oder Europa, S. 512.

${ }^{6}$ Novalis versteht die Aufklärung vor allem als einen Bildungsprozess. Er charakterisiert das Aufklärungsprogramm (die Erfüllung aller Veranlagungen der Menschheit) und seine empirischen (geschichtlichen) Realisierungen, besonders die französische und die deutsche Aufklärung im achtzehnten Jahrhundert. Er weist auf die geschichtlichen Ursachen der Differenz zwischen der französischen und deutschen Aufklärung (besonders die Reformation und die Französische Revolution) hin. Zuletzt beschreibt er die Spuren der neuen höheren Stufe der Aufklärungsgeschichte in Deutschland (die transzendentale
} 
was den Inhalt beider Varianten ausmacht und woraus die erwähnten Unterschiede resultieren. Bei der Beschreibung der Phänomene, die den französischen Geist charakterisieren, scheut Novalis nicht vor Metaphern, die einen Wertsinn haben, wie z. B. „kaltes, todtes Spitzbergen des Stubenverstandes” , „fürchterlicher Wahnsinn”, „weltlicher Protestantismus”, „kluge Aufklärungspläne”, „triumphirende Gelehrsamkeit". Die Einseitigkeit der französischen Aufklärung bestand in dem engen Verständnis des Ziels der Geschichte - da man als übergeordneten Wert den Verstand anerkannte, musste es sich ungünstig in der Ästhetik, Moral- und Gesellschaftstheorie, Religion, Kunst und Philosophie auswirken. Es war eine Erziehung zum Verstand, nicht zu einer allseitigen, „schönen” Menschheit, die die Quintessenz aller Kräfte und Begabungen des Geschlechts darstellen sollte, so auch des Sinnes für Ästhetik, Religion, Gefühl, Willen, Phantasie u.v.a. Nach Schillers Bezeichnung könnte man hier von der Tyrannei der Regeln über dem Gefühl sprechen, was den Zustand der Barbarei bestimmte, der ebenso gefährlich war, wie der ihm gegenübergestellte der Wildheit ${ }^{7}$.

Novalis sieht ein Paradoxon darin, dass die Philosophie der französischen Aufklärung, die die Toleranz (deklarativ) für ihr Synonym erachtete, trotzdem im Grunde genommen äußerst intolerant war gegenüber jeder anderen Religion, als der von ihr selbst aus Wissensfragmenten konstruierte Glaube an Priester und Mistagogen der Aufklärungsidee ${ }^{8}$. Intolerant war sie auch gegenüber der einseitig als eine Etappe der Fehler und des Aberglaubens betrachtete Tradition. Die sog. Philosophen zählten ja zur neuen Philosophie ,alles dazu was dem Alten entgegen war" . Sie verneinten Mannigfaltigkeit und Differenziertheit der Modelle des Gesellschaftslebens, der Ästhetik usw. und arbeiteten daran, „die Welt alles bunten Schmucks zu entkleiden”10. Der normative Verstand sollte in allen Sphären der menschlichen Kultur Richter sein, die Beherrschung der Natur und Gesellschaft durch den Menschen ermöglichen. Gleichzeitig aber, wieder nach Novalis, „setzte [diese Philosophie - H.J.] den Menschen in der Reihe der Naturwesen mit Noth obenan"11. Wiederum paradox haben die Revolutionsereignisse bewiesen, wie sich

Philosophie von I. Kant und J. G. Fichte, die klassische Poesie von J. W. Goethe und F. Schiller, die geschichtliche Philosophie von F. Schlegel).

${ }^{7}$ Siehe F. Schiller, Listy o estetycznym wychowaniu czlowieka i inne rozprawy, übers. von I. Krońska, J. Prokopiuk, Wydawnictwo Czytelnik, Warszawa 1972.

${ }^{8}$ Novalis, Schriften, Bd. III, Christenheit oder Europa, S. 515.

${ }^{9}$ Siehe oben.

${ }^{10}$ Ebenda, S. 4.

${ }^{11}$ Ebenda, S. 515. 
die aufklärerische Ordnungsidee in der Sozialpraxis in das Element unbändiger Anarchie verwandelt, des anderen Zustandes, der eine Entartung des Gesellschaftszustandes darstellt. Ein solches Ergebnis war unabwendbar, denn die Zöglinge der Philosophie der französischen Aufklärung haben die Bildung der eigenen Persönlichkeiten vollständig vernachlässigt und gerade die in dieser Sphäre entstandenen Missverhältnisse mussten zu fürchterlichen politischen Phänomenen an der Jahrhundertwende beitragen. Denn nach Novalis' Überzeugung „das Ideal der Sittlichkeit hat keinen gefährlichen Nebenbuhler als das Ideal der höchsten Stärke - des kräftigen Lebens [...]. Es ist das Maximum des Barbaren - und hat leider in diesen Zeiten der verwildernden Kultur gerade unter den grössesten Schwächlingen sehr viele Anhänger erhalten"12. Deswegen schreibt er, dass jeder Zeitgenosse der Revolution deren Ursachen in sich selbst suchen soll, in seiner eigenen inneren Armseligkeit, in der Vernachlässigung der Realisierung der schönen Menschheit ${ }^{13}$. Das Programm der Selbstbildung, der Selbsterziehung zur echten Mündigkeit, ist also die Voraussetzung des Programms der Beherrschung der Umwelt, der Anpassung dieser Welt an eigenen Willen. Sonst bleibt der Mensch in der Knechtschaft zufälliger Reize aus der Außenwelt und täuscht sich lediglich, dass er die Macht über die Welt besitzt. Diese Täuschung war es, die sich in dem Streben der Revolutionäre nach der Modellierung der Geschichte und Beeinflussung ihrer Richtung äußer$\mathrm{te}^{14}$.

Novalis dagegen ist der Meinung, dass der Geschichtsprozess eine organische Wirklichkeit ist, die durch eigene immanente Gesetze regiert wird. Erst die Erkenntnis und Achtung historischer Gesetzmäßigkeiten ermöglicht wirksames Handeln, aber zu einem solchen Bewusstsein kommt man durch fehlerhafte Tendenzen, die sozusagen Experimente der Geschichte sind, die dann allmählich den eigenen, auf alle menschlichen Dispositionen gestützten Plan erkennt. Das Scheitern der Revolutionäre hatte ihren Ursprung darin, dass die im Geiste der Aufklärungsphilosophie Erzogenen gegen die Geschichtsrichtung ein abstraktes Königreich des Verstandes oder vielmehr das des Wohlstands errichten wollten, in dem jeder Teilnehmer seine Habgier befriedigen könnte und aus der Summe dieser Egoismen sollte eine Gesellschaftsordnung entstehen. Es war eine Art neuer Religion, die den Nutzen für den Götzen der Epoche hielt, gemäß dem primitiven Geist, dem beschränkten

\footnotetext{
${ }^{12}$ Derselbe, Schriften, Bd. II, Vorarbeiten zu verschiedenen Fragmentsammlungen, S. 232.

${ }^{13}$ Derselbe, Schriften, Bd. III, Randbemerkungen zu F. Schlegels Ideen, S. 490.

${ }^{14}$ Derselbe, Schriften, Bd. III, Christenheit oder Europa, S. 518.
} 
Spießbürgerhorizont. Novalis stellt die Frage, ob die Revolution französisch bleiben soll, so wie die Reformation eine deutsche Leistung war. Daraus können wir schließen, dass er historische Ursachen der Differenz zwischen französischen und deutschen Varianten der Aufklärung erkannt hat. Beinahe nach Hegels Art und Weise entscheidet er, das eine Revolution ohne Reformation d. h. ohne Befreiung des Gewissens unmöglich ist. Da aber in Frankreich diese Voraussetzung nicht erfüllt worden war, zeugten Revolutionsereignisse von der Krise der verfrühten Reife, die aus dem Fehlen der Freiheit resultierte ${ }^{15}$.

Nach Novalis' Überzeugung hat jede Nation, genauso wie jedes Individuum, eigene Lehrjahre, die über ihre Spezifik und Einmaligkeit sowie über die unterschiedlichen Realisierungsvarianten des Aufklärungsprogramms entscheiden. So hält Novalis die „neue französische Manier” (,gemeine, prosaische Aufklärung”) dem deutschen „Stil”16 („wahre, höhere, poetische Aufklärung") entgegen. Versuchen wir nun zusammenzufassen, woraus die „französische Manier”, diese eingeengte Aufklärungsvariante besteht. Sie wird von dem sog. Philisterbewusstsein vertreten, das den menschlichen Geist den Gesetzen der Mechanik unterwirft. Demnach ist es nicht imstande, die Freiheitsidee zu begreifen und gravitiert zum Fatalismus. Seine nächste Sünde ist der Versuch, jegliche Mannigfaltigkeit auszurotten, die Satuierung eines einzigen Gesetzbuches des Schönen und Wahren für alle Epochen und Nationen, was die Unterschätzung solcher Werte bedeutet, wie Nationalität, Originalität und Toleranz ${ }^{17}$. Sein Merkmal ist eine gewisse Ungeduld, die sich im Wunsch äußert, in eine neue Etappe der Geschichte überzuspringen, die vom räsonierenden Intellekt vorprogrammiert, dadurch als postulativ und ahistorisch ist $^{18}$. Es rechtfertigt egoistische Einstellungen, weil es sich für den Utilitarismus ausspricht. Seinen engen, beschränkten, partikulären Gesichtspunkt projiziert es auf das Verständnis der Sozialbindung und führt in der Praxis zur politischen Quadratur des Kreises, zu den Entartungen des Gesellschaftszustands ${ }^{19}$. Es ist einseitig in seiner

\footnotetext{
${ }^{15}$ Derselbe, Schriften, Bd. II, Vermischte Bemerkungen, S. 116, Blütenstaub, S. 105.

${ }^{16}$ Die Unterscheidung „Manier” und „Stil” erscheint in der Diskussion über das Übersetzungsprogramm der Antike. Bei Novalis wird sie auf die Unterschiede zwischen der französischen und deutschen Kultur bezogen. Siehe derselbe, Schriften, Bd. II, Vermischte Bemerkuingen S. 26, Blütenstaub, S. 25, Vorarbeiten zu verschieden Fragmentsammlungen, S. 56, Bd. III, Glauben und Liebe S. 23; auch in den Briefen, Bd. IV, an Reinhold 5.10.1791, S. 95-96, an Schiller 7.10.1791, S. 100.

${ }^{17}$ Derselbe, Schriften, Bd. IV, an F. Schlegel 7.10.1791, S. 99.

${ }^{18}$ Ebenda, Bd. IV, an R. Just 5.12.1798, S. 266.

${ }^{19}$ Derselbe, Schriften, Bd II, Glauben und Liebe, S. 36.
} 
Verherrlichung des Stubenverstandes und in seiner Aggression gegen die anderen Geisteskräfte des Menschen, was eine krüppelhafte, disharmonische Verwirklichung der Menschheit bewirkt ${ }^{20}$. Seinen historisch bedingten Vorurteilen schreibt es den Rang allgemeingültiger und universeller Wahrheiten. Es glaubt seine Reife erreicht zu haben, indes es in der Tat eine „Krise der eintretenden Pubertät” repräsentiert ${ }^{21}$. Seine Errungenschaften und Niederlagen waren aber eine Geschichtslektion für die deutschen Intellektuellen.

Trotzdem also die Lehrjahre der einzelnen Nationen unterschiedlich sind, können aus den Erfahrungen und Fehlern des jeweiligen Landes die anderen profitieren und diese in eigene, der Originalität nicht beraubte Denkkonzepte verwandeln. Novalis meint damit offensichtlich eine produktive Assimilation des fremden Erbes nach dem Prinzip, dass das Gegebene kein Dogma ist, sondern vielmehr ein Reiz zur eigenen Selbstständigkeit ${ }^{22}$. Er bemerkt auch, das im Streben nach der Verwirklichung der Werte die Führenden ihre Plätze wechseln, Meister erneut $\mathrm{zu}$ Lehrlingen werden und die Lehrlinge zu Meistern ihrer bisherigen Meister und Schutzherren ${ }^{23}$. So hat auch die deutsche Kultur die Errungenschaften des französischen Geistes überholt, weil gerade auf ihrem Boden die Entdeckung der eigenartigen Wirkungsmethodik erfolgen konnte, die die Möglichkeit aufzeigt, dass jedes Individuum fähig ist, die eigene Naturgeschichte in Bildungsgeschichte i.e. Aufklärungsgeschichte $\mathrm{zu}$ verwandeln ${ }^{24}$. Dagegen war in der französischen Philosophie, die in ihrem Charakter naturalistisch ist, ein solcher Umbruch unmöglich. Ihre Vertreter stellten darüber hinaus dogmatisch einen starren Gegensatz zwischen dem gebildeten und ungebildeten Teil der jeweiligen Gesellschaft, auch innerhalb Europas und mussten sich das Recht an, andere $\mathrm{zu}$ ihrem Glauben $\mathrm{zu}$ bekehren ${ }^{25}$. So entstand die Situation, dass nicht die Autorität der Tradition, des Brauches anerkannt werden musste, sondern die des neuen Erziehers-Philosophen. Daraus folgte als Kon-

${ }^{20}$ Derselbe, Schriften, Bd. III, Fragmente und Studien, S. 291.

${ }^{21}$ Derselbe, Schriften, Bd. II Vermischte Bemerkungen S. 116, Blütenstaub, S. 105.

${ }^{22}$ Derselbe, Schriften, Bd. II, Vorarbeiten zu verschiedene Fragmentsammlungen, S. 66.

${ }^{23}$ Siehe oben, S. 3.

${ }^{24}$ Derselbe, Schriften, Bd. II, Vermischte Bemerkungen, S. 93, Blütenstaub, S. 94.

${ }^{25}$ Die Opposition Ungebildete-Gebildete bedeutete die Aussonderung der Unterschicht und Oberschicht und war mit der abschätzenden Beurteilung des Volkes verbunden, als eines Synonym der Grobheit und Ignoranz. P. Kluckhohn macht darauf aufmerksam, dass im 18. Jh. der Begriff „Nation” umfassender war als „Volk”. Ausserdem stellt er fest, dass man bereits bei Herder und später bei den deutschen Romantikern eine hohe Wertung des Volksglaubens und der Volkskunst findet. Siehe P. Kluckhohn, Das Ideengut der deutschen Romantik, Niemeyer, Halle 1941, S. 97-98. 
sequenz (die in der utilitaristischen Philosophie noch verstärkt war, weil dort der Mensch als ein Produkt der Erziehung gesehen wurde) trotz allem die Vormundschaft über die Mehrheit der Gesellschaft, was der Idee der Mündigkeit, Selbständigkeit und Selbsterziehung widersprach. Novalis bemerkt ironisch, das in der Neuzeit, wie die Geistlichen im Mittelalter, die Zunft der Aufklärer und Philanthropen Vormünder $\operatorname{sind}^{26}$. Er vergleicht es mit der alttestamentarischen Denkweise, indem er mit Bedauern feststellt: "Das Neue Testament ist uns noch ein Buch mit 7 Siegeln" 27.

Er kritisiert die Beschränktheit der Verwirklichung des Aufklärungsprogramms, diese erstarrte Fassung einer ehrgeizigen Zielsetzung, die Mündigsprechung sein sollte. Dabei versucht er, als höchsten Wert die Autonomie jedes Individuums, jeder Nation usw. zu verteidigen. Das erfordert eine Distanzierung von den Vorurteilen der Modephilosophen der französischen Aufklärung - vom mechanistischen Determinismus, Naturalismus und Sensualismus. So sehen wir, dass Novalis einerseits die Aufklärungsformation in der französischen Fassung beurteilt (gemeine Aufklärung), andererseits aber der Aufklärungsidee einen normativen und programmatischen Sinn gibt. Der Kantschen Inspiration entnimmt er die Überzeugung, dass jeder Mensch sein eigener Gesetzgeber $d$. h. ein autonomes Wesen werden soll, das keinen äußeren Determinanten unterliegt ${ }^{28}$. Die Perspektive einer realisierten höheren Aufklärung bedeutet - nach Novalis - einen Zustand, in dem jedes Individuum begreift, dass die Aufklärung seines eigenen Intellekts mehr Nutzen bringt als alle äußeren Rezepte ${ }^{29}$.

Dies könnte man als eine Fortsetzung des Aufklärungsgedankens betrachten, die konsequenteste, die erst die deutschen Denker geleistet haben. Diesen Gedanken veranschaulicht zutreffend ein Dialogfragment, in dem Novalis behauptet, man solle nicht abwarten, sondern die Erfüllung der Idee der Menschheit suchen. Um diese Überzeugung zu betonen, stellt er zwei Gesprächspartner gegenüber, von denen einer meint, „,er Kranke lässt den Arzt rufen, weil er sich nicht helfen kann”, der andere dagegen erwidert: „Wenn nun aber der Arzt gerade zur Arz-

\footnotetext{
${ }^{26}$ Novalis, Schriften, Bd III., Christenheit oder Europa, S. 516.

${ }^{27}$ Derselbe, Schriften, Bd III., Fragmente und Studien, S. 84.

${ }^{28}$ Novalis akzeptiert den Kantschen Gegensatz zwischen Autonomie und Heteronomie. Er weiß, dass die Autonomie die innere Gesetzgebung der praktischen Vernunft bedeutet. Nach Novalis Meinung soll jeder Mensch ein moralisches, selbständiges und aktives Wesen werden. Siehe derselbe, Schriften, Bd II., Kant-Studien, S. 49; Bd. III, Das allgemeine Brouillon, S. 460, 463.

${ }^{29}$ Derselbe, Schriften, Bd. III, Das allgemeine Brouillon, 1131.
} 
ney dem Kranken Anstrengung seines Verstandes verschreibt. Wer sich selbst fehlt, kann nur dadurch geheilt werden, dass man ihm sich selbst verschreibt" ${ }^{\prime 30}$. Daraus ist zu schließen, dass man niemandem Reife schenken kann, dass man auch die Errungenschaften des reifen Alters nicht exportieren darf, wo sich das Individuum auf einer anderen Entwicklungsstufe befindet, wie es die französischen Revolutionäre versuchten, die sich für die Missionäre Europas hielten. Die Aufklärung soll im Prinzip Selbstaufklärung, Selbsterziehung, Selbstbildung sein. Es gibt kein allgemeines Naturgesetz, das automatisch deren Realisierung garantieren und somit den Menschen von seiner aktiven Beteiligung am Geschichtsprozess befreien könnte. Dieser Fehler französischen Ideologen erinnert Novalis an den alten Fehler des Judentums - Warten auf den Messias, der radikal ein neues Königsreich der Gerechtigkeit errichten sollte ${ }^{31}$. Indessen erfolgt die Realisierung einer vollkommenen Aufklärung, einer vollkommenen Bildung nach seiner Überzeugung allmählich in der natürlichen Geschichte jedes Individuums; damit ist die Ungeduld aller Schwärmer unbegründet, die in der Opposition zur bisherigen Geschichte eine neue Welt errichten möchten, eine Welt ohne Präzedenzfälle in der Tradition, die als Sphäre der Fehler und Vorurteile verurteilt ist ${ }^{32}$. So war es nicht das 18. Jahrhundert, das den Fortgang des Menschen von der von ihm verschuldeten Unmündigkeit bewirkt hat, denn die Vorgängerelemente dieser Idee waren bereits auf früheren Etappen vorhanden und konnten erst nach den Erfahrungen der Revolutionsepoche begriffen werden.

\section{Die Bedeutung der Reformation für die Realisierung der Aufklärungsidee in Deutschland}

Von besonderer Bedeutung für die Realisierung der Aufklärungsidee war nach Novalis die Reformation. Sie begründete nämlich die Autonomie des Gewissens jedes Gläubigen und erklärte zum Unrecht jede äußere Vormundschaft eines getrennten Geistlichenstandes. Die Vormundschaft an sich ist noch nicht Schlechtes, sie kann sogar notwendig und wohltätig auf einer bestimmten Entwicklungsstufe sein, muss aber so Novalis - überprüft werden, wenn sie zu einem überholten Zwang und zur Fortschrittsbremse wird. Und obwohl in der Geschichte des Protestantismus viele unvorhergesehene und für das Gesellschaftsleben negative Konsequenzen zum Vorschein gekommen sind, war es doch

\footnotetext{
${ }^{30}$ Derselbe, Schriften, Bd. II, Dialogen, S. 666.

${ }^{31}$ Ebenda, S. 662.

${ }^{32}$ Derselbe, Schriften, Bd. III, Christenheit oder Europa, S. 518.
} 
ein Anfang der Selbstbefreiung des Menschen von der Macht des Positi$v^{3} n^{33}$. Daher erhielt im protestantischen Deutschland die Philosophie einen mächtigen Anstoß, die in diesen Ländern nie so radikal mit dem Religionserbe abbrach, wie es etwa im aufklärerischen Frankreich der Fall war. Man könnte sagen, dass Deutschland die bekannten Religionsvorstellungen in die Sprache der Philosophie übertragen hat, während Frankreich Erbe des Protestantismus im Bereich der Politik war, in diesen Anwendungsbereichen also, die eher vom verhängnisvollen Buchstaben als vom Geist bestimmt waren. Bei der Charakteristik der französischen Aufklärung schreibt Novalis, dass deren Vertreter, der Macht der „Gelehrsamkeit” vertrauend, den Glauben für die Ursache der allgemeinen Stagnation erklärt haben.

Die deutsche Aufklärung dagegen präsentierte eine völlig andere Einstellung, deswegen stellt Novalis fest: „In Deutschland betrieb man dieses Geschäft gründlicher, man reformierte das Erziehungswesen, man suchte der alten Religion einen neuen vernünftigen gemeinern Sinn zu geben, indem man alles Wunderbare und Geheimnisvolle sorgfältig von ihr abwusch; alle Gelehrsamkeit ward aufgeboten um die Zuflucht zur Geschichte abzuschneiden, indem man die Geschichte zu einem häuslichen und bürgerlichen Sitten- und Familien-Gemählde zu veredeln sich bemühte" ${ }^{34}$. Dieser Unterschied hat darüber entschieden, dass die zweite Reformation unumgänglich war, und dass sie - so Novalis das Land heimsuchen musste, in dem die Befreiung des Gewissens nicht erfolgt war. Dadurch u.a. wurde die Philosophie in absolute Opposition zum Glauben gestellt. Novalis missbilligt auch die Versuche, in der deutschen Aufklärung die Religion $\mathrm{zu}$ rationalisieren. Mit Ironie spricht er von den klugen Köpfen der Neologen, die über die Buchstaben diskutieren und zur Intoleranz gegenüber sog. Atheisten neigen, die wie Spinoza oder Fichte eher eine pantheisierende Einstellung vertre$\operatorname{ten}^{35}$. Mit dieser Einstellung gerade verbindet er den Glauben an die moralische Weltordnung, an das unendliche Menschengeschlecht, d.h. an den pantheistisch erscheinenden Gott bzw. Natur. Der so begriffene Glaube ermöglicht es, nach Novalis, die ganzheitliche Fassung mit der individualisierenden in Einklang zu bringen. Manchmal heißt es bei ihm Geist der Christenheit. Die Aufklärung, die eine solche Einstellung verurteilte, musste sich mit der eigenen Dämonie begnügen, mit der Auf-

\footnotetext{
${ }^{33}$ Ebenda, S. 517.

${ }^{34}$ Ebenda, S. 516.

${ }^{35}$ Siehe derselbe, Schriften, Bd. II, Kann ein Atheist auch moralisch und tugendhaft aus Grundsätzen seyn?, S. 19. Auch Bd. III, Das allgemeine Brouillon, 1098.
} 
fassung der Welt als eines perpetuum mobile. Oder aber beim Versuch der Rationalisierung der Religion - einem nüchternen, enthusiasmusfeindlichen Denken - betrieb sie eine Theologie der historisch-kritischen Vernunft, die die Glaubwürdigkeit der Bibel beweisen sollte. Diese Aufgabe war selbstverständlich etwas anderes als der Weg des eigenen Gewissens, das die Handlungsrichtung ohne jegliche äußere Vormundschaft festlegte $^{36}$. Über die Vertreter des nüchternen Denkens behauptet Novalis, sie realisieren einseitig unendlich unterschiedliche Dispositionen zur Menschheit, unterordnen sich zu leicht und kritiklos den in ihrer Epoche verbreiteten Bräuchen, Gewohnheiten und Vorurteilen ${ }^{37}$. Sie seien bereit, die Grenzen der eigenen Geistesformation an alles Andersartige anzupassen, an alles, was schöpferische Mühe beim Verständnis der Geschichte erfordert, die man mit einer Reihe vergleichen kann, die durch variable, trotzdem aber miteinander verbundene Faktoren konstituiert wird. Daher spricht Novalis von den Philosophen der französischen Aufklärung als von „Modephilosophen”38, „Philosophen der Beschränkung" 39 und von einem, nämlich Voltaire, formuliert er die Bemerkung" „sein Candide ist eine Odyssee”, ,seine Welt ein Pariser Boudoir”

Eine Folge der Tätigkeit der Aufklärungsdenker war - nach der Meinung Novalis' - die Tatsache, dass die Einrichtung der Kritik, einst gegen die alten Autoritäten gerichtet, sich in der Geschichtsdialektik in eine Kritik der Kritik verwandeln musste, d. h. in die Kritik des Verstandes selbst" ${ }^{\prime 1}$. Den Angriff auf die Repräsentanten der Religion, gemeinsam von Gelehrten und Regierenden geführt, verwandelte sich in einen Angriff auf die Regierung, die für eine erstarrte Gesellschaftsordnung erachtet worden ist, die allen Forderungen des Verstandes trotzt. Dann musste aber der gesetzgebende Verstand seine eigene Niederlage in Kauf nehmen, die aus der fehlenden Respektierung des Geschichtsprozesses resultierte. Die Natur erwies sich als Wirklichkeit, die sich einer so radikalen und willkürlichen Modernisierung nicht unterziehen ließ, wie es die Philosophen erwarteten, die vom Stubenverstand geformt waren. „Kluge Aufklärungs-Pläne” erreichten ihre Erschöpfung in einer wahren Anarchie der Revolutionsereignisse und führ-

\footnotetext{
${ }^{36}$ Derselbe, Schriften, Bd. IV, an Just, 26.12.1793, S. 272.

${ }^{37}$ Derselbe, Schriften, Bd. I, Heinrich von Ofterdingen, S. 326.

${ }^{38}$ Derselbe, Schriften, Bd. II, Vorarbeiten zu verschiedene Fragmentsammlungen, S. 117.

${ }^{39}$ Derselbe, Schriften, Bd. II, Vermischte Bemerkungen S. 103, Blütenstaub, S. 113.

${ }^{40}$ Derselbe, Schriften, Bd. II, Vorarbeiten zu verschiedene Fragmentsammlungen, S. 56.

${ }^{41}$ Derselbe, Schriften, Bd. III, Christenheit oder Europa , S. 529. Siehe das interessante Kapitel: Kritik der Zeit und Kritik der Kritik, [in:] H. Schanze, Romantik und Aufklärung. Untersuchungen zu Friedrich Schlegel und Novalis, Carl, Nürnberg 1966.
} 
ten letzten Endes zur Umkehrung. Diese Krise spielte die Rolle eines Tiegels, in dem, wie in einem chemischen Prozess, aus der Mischung zweier Komponenten eine dritte entsteht, die aber keine einfache Summe von beiden ist ${ }^{42}$.

Die Revolution interpretierte Novalis als den Beginn ein wahren, inneren Revolution ${ }^{43}$, eine höheren Aufklärung, deren Bedingungen zu verstehen nicht nur die französische, aber auch die deutsche Aufklärung nicht imstande war, die beide außer Unterschieden auch viele dem „räsonierenden Verstand” gemeinsame Momente enthielten. Daher spricht er sowohl an die französische Revolutionäre, als auch an diejenigen deutschen Denker, die das Vorbild der Republik spießbürgerlich in der französischen Manier erblicken, die bedeutenden Worte aus: „an der Geschichte verweise ich euch"44. Er drückt darin die eigene Überzeugung aus, dass es an der Zeit ist, den philosophischen Streit um den Buchstaben und das Vorteil aufzugeben, dass die bisherige Geschichte ein Fehlerbereich und eine starke Antithese der Wahrheit war. Man solle die Versuche aufgeben, die Geschichte nach Dekreten des aufgeklärten Verstandes zu modellieren, man solle mit der Verachtung des Glaubens brechen, der einzig imstande ist, das beschränkte Denken von den Ketten des Partikularismus zu befreien und die universalistische mit der individualisierenden Tendenz zu versöhnen.

\section{Die neue höhere Stufe der Aufklärungsgeschichte in Deutschland}

Novalis weist darauf hin, dass „erste Spuren einer neuen Welt” in Deutschland beobachten könne, das eine qualitative neue Entwicklungsetappe der Kultur eröffne. Er scheint damit die Philosophen zu meinen, die auf Grund der behandelten Thematik zur Aufklärung gezählt werden könnten, die aber durch ihre neuen, schöpferischen Entscheidungen das Aufklärungsparadigma bereits überschritten haben. Sie beginnen, um mit Novalis zu sprechen, die Etappe der „höheren”, „wahren Aufklärung”. Ein Beweis dafür sei die Transzendentalphilosophie, die die Leere des flachen Empirismus und leeren Rationalismus meidet. Entgegen der Auffassung, die die Determinierung des Menschen durch seine Umwelt verkündet, begründet diese Philosophie eine andere Gesetzgebung - die

\footnotetext{
${ }^{42}$ Derselbe, Schriften, Bd. II, Dialoge, S. 667.

${ }^{43}$ Siehe R. Brinkmann, Deutsche Frühromantik und Französische Revolution, [in:] Deutsche Literatur und Französische Revolution, Vandenhoeck\&Ruprecht, Göttingen 1974.

${ }^{44}$ Novalis, Schriften, Bd. III, Christenheit oder Europa, S. 518.
} 
der praktischen Vernunft, der den Menschen als ein moralisches, selbständiges und aktives Wesen bestimmt. Kant z. B. unternimmt die Kritik aller menschlichen Kräfte, indem er die Beschränktheit der theoretischen Vernunft und die Perspektive aufzeigt, über diese Grenzen hinaus in die Sphäre der Metaphysik der Sitten zu gelangen. In Anlehnung an den Kantschen Anstoß gründet Fichte eine - nach der Einschätzung der Romantiker $^{45}$ - revolutionäre Wissenschaftslehre, die in ihrer Polemik mit dem Naturalismus die Menschenwürde mit der autonomen Aktivität des Menschen begründet, die in der permanenten Überwindung des eigenen Nicht-Ich, d. h. des Passivismus besteht ${ }^{46}$. Die Antinomien der Aufklärung weichen allmählich den dialektischen Beschreibungen der Geschichte des reinen Denkens und der realen Welt. Wohl in der Entstehung dieser Antinomien erblickt Novalis das Verdienst der Aufklärungsgelehrten, wenn er schreibt:"dieser Wahn musste zum Besten der Nachkommen erschöpft" ${ }^{\prime 7}$ werden. In den Transzendentalismustheorien bemerkt er darüber hinaus die Möglichkeit, den philosophischen Unterbau für das politische Leben zu finden. Das begründet die Überzeugung, dass der Utilitarismus dieses Problem nicht gelöst hat. So bemerkt Novalis im zeitgenössischen deutschen Denken eine große Ladung an Originalität und Innovation.

Für die Deutschen spricht auch die Tatsache, dass sie historische Interessen erweckt haben. Winckelmann war Entdecker der Schönheit der antiken Skulptur, wie F. Schlegel der Poesie der Griechen und Römer und Novalis selbst der Rolle des Christentums im Geschichtsprozess $^{48}$. Große Verdienste hatte die deutsche Literatur auf dem Gebiet der Übersetzung: dank L. Tieck wurde Cervantes entdeckt, dank A. W. Schlegel - Shakespeare. Das veranlasst Novalis $\mathrm{zu}$ folgender übermütiger Bemerkung: „Außer den Römern sind wir einzige Nation, die den Trieb des Übersetzens so unwiderstehlich gefühlt, und ihm so unendlich viel Bildung schuldig sind" ${ }^{49}$, und an einer anderen Stelle: „Das Beste, was die Franzosen in der Revolution gewonnen haben, ist eine Portion Deutschheit" ${ }^{\prime 50}$. Novalis meint, dass interessante Ände-

\footnotetext{
${ }^{45}$ F. Schlegel, Ideen, [in:] Athenaeum. Eine Zeitschrift von August Wilhelm Schlegel und Friedrich Schlegel, Bd. I, S. 56.

${ }^{46}$ Novalis, Schriften, Bd. III, Das allgemeine Brouillon, S. 463.

${ }^{47}$ Derselbe, Schriften, Bd. III, Christenheit oder Europa, S. 520.

${ }^{48}$ Es handelt sich um folgende Arbeiten: J. Winckelmann, Geschichte der Kunst, 1764; F. Schlegel, Über das Studium der griechischen Poesie, 1795; F. Schlegel, Geschichte der Poesie der Griechen und Römer, 1798; Novalis, Christenheit oder Europa, 1799.

${ }^{49}$ Novalis, Schriften, Bd. IV, an A. W. Schlegel 30.11.1797, S. 273.

${ }^{50}$ Novalis, Schriften, Bd. II, Vermischte Bemerkungen, S. 63, Blütenstaub, S. 64.
} 
rungen auch im Bereich der Religion zu verzeichnen sind, u. a. dank Schleiermacher, der festgestellt hat, dass die wahre Religion darin besteht, der Menschheit individuell darzulegen, was der polemische Akzent gegenüber dem die Mannigfaltigkeit nivellierenden Aufklärungsgedanken enthält ${ }^{51}$. Ein anderes Symbol der Möglichkeit des deutschen Geistes sind für Novalis Goethe und Schiller, die Erzieher des kommenden Jahrhunderts genannt werden ${ }^{52}$.

Man könnte die Verallgemeinerung wagen, dass die Geburt der neuen deutschen Kultur um die Dämmerung der Aufklärung dank der Generation junger Intellektueller erfolgte, die sich durch vorurteilslose Öffnung auf verschiedene Inspirationen auszeichnete. Absorptive Einstellung ist wie es die Metapher des ungehemmten, ungebundenen Kindergeistes beweist - ein Merkmal der genialen Persönlichkeit, eine Antithese der Einseitigkeit und Erstarrung. Dieses Bild kann per analogiam auf die Entwicklung der Nationalkulturen bezogen werden, denn, nach Novalis, die Nation ist wie das Kind ein pädagogisches, individuelles Problem. Nach seiner Überzeugung kann eine von den anderen isolierte Begabung früh ihren Höhepunkt erreichen, sie verblüht aber auch früh ${ }^{53}$. Diese Behauptung kann man auf die einseitig auf Verstand ausgerichtete französische Philosophie beziehen. Deswegen schreibt er von ihren Vertretern „vorzeitig Gereifte”, was in diesem Kontext bereits abfällig beurteilte Erstarrungsetappe dieser Formation bedeutet. Erst die Entwicklung aller ergänzenden Talente würde „die Spuren einer neuen Welt” aufweisen, zu der man erst reif werden soll, indem man aus den bisherigen Geschichtsversuchen immer deutlicheres Erkennen ihres Plans lehrnt. Gerade der Geschichtssinn führt Novalis zur folgenden Schlussfolgerung: [In Deutschland - H. J.] „eine gewaltige Ahnung der schöpferischen Willkühr, der Grenzenlosigkeit, der unendlichen Mannigfaltigkeit, der heiligen Eigenthümlichkeit und der Allfähigkeit der inneren Menschheit scheint überall rege zu werden. Aus dem Morgentraum der unbehülflichen Kindheit erwacht, übt ein Theil des Geschlechts seine erste Kräfte an Schlangen, die seine Wiege umschlingen und den Gebrauch seiner Gliedmassen ihm benehmen wollen"54. Dies bedeutet die Befreiung von den Vorurteilen der sog. gemeinen Aufklärung und dieser Prozess erfolgt durch Selbstpolemik, Selbsterleuchtung,

\footnotetext{
${ }^{51}$ Derselbe, Schriften, Bd. III, Christenheit oder Europa, S. 521.

${ }^{52}$ Derselbe, Schriften, Bd. IV, an Reinhold 5.10.1791, S. 95. Siehe auch Bd II, Vorarbeiten zu verschiedene Fragmentsammlungen, S. 54, 445, Vermischte Bemerkungen, S. 117-118, Blütenstaub, S. 106.

${ }^{53}$ Derselbe, Schriften, Bd. III, Das allgemeine Brouillon, S. 306.

${ }^{54}$ Derselbe, Schriften, Bd. III, Christenheit oder Europa, S. 519.
} 
Selbstkritik des deutschen Geistes. Novalis suggeriert, dass man eine Verbreitung dieses höheren Bewusstseins unter den Nationen Europas und dann anderer Weltteile nicht erwarten soll. Dieses Ziel kann nicht durch aufdringliches Wiederkehren von außen realisiert werden, sondern durch das Anregen jedes Individuums zur eigenen Selbständigkeit. Es ist schwer zu erraten, welche weiteren Inhalte und Werte in der späteren, noch nicht vollendeten Aufklärungsgeschichte entdeckt werden können. Novalis spricht lediglich von den Spuren einer neuen Welt, da er überzeugt ist, dass man die Zukunft vollständig und mit Einzelheiten nicht dekretieren kann.

\section{Literarturverzeichnis}

Athenaeum. Eine Zeitschrift von August Wilhelm Schlegel und Friedrich Schlegel, Bd. I-III, Berlin 1798-1900, Nachdruck Darmstadt 1983.

Behler E., Frühromantik, Walter de Gruyter, Berlin 1992.

Beiser F. C., Enlightenment, Revolution and Romanticism, Harvard University Press, Cambridge 1992.

Beiser F. C., The Romantic Imperative: The Concept of Early German Romanticism, Harvard University Press, Cambridge 2003.

Blütenstaub. Rezeption und Wirkung des Werkes von Novalis, hrsg. von $\mathrm{H}$. Uerlings, Max Niemeyer Verlag, Tübingen 2000.

Brinkmann R., Deutsche Frühromantik und Französische Revolution, [in:] Deutsche Literatur und Französische Revolution, Vandenhoeck \& Ruprecht, Göttingen 1974.

Frank M., Unendliche Annäherung. Die Anfänge der philosophischen Frühromantik, Suhrkamp Verlag, Frankfurt am Main 1997.

Haering Th., Novalis als Philosoph, W. Kohlhammer Verlag, Stuttgart 1954.

Herder J. G., Auch eine Philosophie der Geschichte zur Bildung der Menschheit, Suhrkamp, Frankfurt am Main 1967.

Jakuszko H., Novalis. Ksztaltowanie się romantycznej filozofii historii, Wydawnictwo UMCS, Lublin 1993.

Kant und die Zukunft der europäischen Aufklärung, hrsg. von H. Klemme, Walter de Gruyter, Berlin 2009.

Kasperowski I., Mittelalterrezeption im Werk des Novalis, Walter de Gruyter, Tübingen 1994.

Kluckhohn P., Das Ideengut der deutschen Romantik, Niemeyer, Halle 1941. 
Novalis, Schriften. Die Werke Friedrich von Hardenbergs, hrsg. von P. Kluckhohn und R. Samuel unter Mitarbeit von H. Ritter, G. Schulz, H. J. Mähl, Dritte, nach den Handschriften ergänzte, erweiterte und verbesserte Auflage, Bd. I-VI (VI/1-3) W. Kohlhammer Verlag, Stuttgart 1960-2006.

Novalis. Beiträge zu Werk und Persönlichkeit Friedrich von Hardenbergs, hrsg. von G. Schulz, Wissenschaftliche Buchgesellschaft, Darmstadt 1970.

Nassar D., The Relevance of Romanticism: Essays on German Romantic Philosophy 1795-1804, University of Chicago Press, Chicago 2014.

Romantik in Deutschland, hrsg. von R. Brinkmann, Metzler, Stuttgart 1978.

Schanze H., Romantik und Aufklärung. Untersuchungen zu Friedrich Schlegel und Novalis, Verlag Hans Carl, Nürnberg 1966.

Schiller F., Listy o estetycznym wychowaniu czlowieka i inne rozprawy, übers. von I. Krońska, J. Prokopiuk, Wydawnictwo Czytelnik, Warszawa 1972.

Schleiermacher F. D. E., Reden über die Religion. Reden an die Gebildeten unter ihren Verächtern, Felix Meiner Verlag, Hamburg 1970.

Schneiders W., Hoffnung auf Vernunft. Aufklärungsphilosophie in Deutschland, Meiner, Hamburg 1990.

Senckel B., Individualität und Totalität. Aspekte zu einer Anthropologie des Novalis, Max Niemeyer Verlag, Tübingen 1983.

Zimmermann H., Aufklärung und Erfahrungswandel. Studien zur deutschen Literaturgeschichte des späten 18. Jahrhunderts, Wallenstein-Verlag, Göttingen 1999.

\section{Summary}

\section{The Rationale Behind the Enlightenment in the Philosophy of Novalis}

Based on original/period texts, the article constitutes the reconstruction of the ways in which Novalis (a representative of the early German Romanticism) understands the term „Enlightenment”. To him, the Enlightenment is coterminous with the historical process of the history of culture (Bildungsgeschichte), the aim of which is the comprehensive development of humanity's holistic abilities and competences. In its narrower sense, the Enlightenment may refer only to the eighteenth century, construed as „the Age of the Enlightenment". In his research, Novalis emphasises the historical contexts that conditioned the differences between the Enlightenment in France and in Germany (among others the German Reformation and the French Revolution). He defines the beginning of the new and higher phase of the German Enlightenment as Kante's and Fichte's Transcendental Philosophy, the Weimar Classicism of Goethe and Schiller as well as Schlegel's Early German Romantic historical philosophy. 
Keywords: Program of Enlightenment, Variant of Enlightenment, Early German Romanticism, Novalis, History of Culture

\section{Streszczenie}

\section{Sensy idei oświecenia w filozofii Novalisa}

Artykuł stanowi - udokumentowaną na tekstach źródłowych - rekonstrukcję sposobu, w jaki Novalis (reprezentant wczesnego romantyzmu niemieckiego) rozumie termin „oświecenie”. Jego zdaniem, oświecenie jest tożsame z procesem historycznym lub historią kultury (Bildungsgeschichte), której celem jest wszechstronny rozwój wszystkich predyspozycji człowieczeństwa. W wąskim znaczeniu oświecenie może być odnoszone do wieku XVIII, określanego jako wiek świecenia. Novalis zwraca uwagę na historyczne przesłanki warunkujące różnicę między oświeceniem francuskim a niemieckim ( $\mathrm{m}$. in. reformację w Niemczech i rewolucję francuską). Za początek nowego i wyższego etapu oświecenia w Niemczech uznaje filozofię transcendentalną I. Kanta i J. G. Fichtego, klasycyzm weimarski J. W. Goethego i F. Schillera oraz filozofię historyczną F. Schlegla -reprezentanta wczesnego romantyzmu niemieckiego.

Słowa kluczowe: program oświecenia, warianty oświecenia, wczesny romantyzm niemiecki, Novalis, historia kultury

Information about Author:

HONORATA JAKUSZKO, habilitated doctor, associate professor, Institute of Philosophy, Maria Curie-Skłodowska University in Lublin, Poland; address for correspondence: Pl. Marii Curie-Skłodowskiej 4, PL 20-031 Lublin. E-mail: honjakuszko@wp.pl 